Article

\title{
Visible Light Induced Green Transformation of Primary Amines to Imines Using a Silicate Supported Anatase Photocatalyst
}

\section{Sifani Zavahir and Huaiyong Zhu *}

School of Chemistry, Physics and Mechanical Engineering, Science and Engineering Faculty, Queensland University of Technology, Brisbane QLD 4001, Australia;

E-Mail: fathimasifani.zavahir@hdr.qut.edu.au

* Author to whom correspondence should be addressed; E-Mail: hy.zhu@qut.edu.au; Tel.: +61-73138-1581.

Academic Editor: Pierre Pichat

Received: 30 October 2014 / Accepted: 22 January 2015 / Published: 26 January 2015

\begin{abstract}
Catalytic oxidation of amine to imine is of intense present interest since imines are important intermediates for the synthesis of fine chemicals, pharmaceuticals, and agricultural chemicals. However, considerable efforts have been made to develop efficient methods for the oxidation of secondary amines to imines, while little attention has until recently been given to the oxidation of primary amines, presumably owing to the high reactivity of generated imines of primary amines that are easily dehydrogenated to nitriles. Herein, we report the oxidative coupling of a series of primary benzylic amines into corresponding imines with dioxygen as the benign oxidant over composite catalysts of $\mathrm{TiO}_{2}$ (anatase)-silicate under visible light irradiation of $\lambda>460 \mathrm{~nm}$. Visible light response of this system is believed to be as a result of high population of defects and contacts between silicate and anatase crystals in the composite and the strong interaction between benzylic amine and the catalyst. It is found that tuning the intensity and wavelength of the light irradiation and the reaction temperature can remarkably enhance the reaction activity. Water can also act as a green medium for the reaction with an excellent selectivity. This report contributes to the use of readily synthesized, environmentally benign, $\mathrm{TiO}_{2}$ based composite photocatalyst and solar energy to realize the transformation of primary amines to imine compounds.
\end{abstract}

Keywords: benzylamine; imine; visible light; anatase; silicate 


\section{Introduction}

Imines are a group of $N$-containing compounds, with a pivotal role as chemically and biologically useful intermediates in various cycloaddition, condensation and reduction reactions [1,2]. These compounds play a major role in pharmacophores, fragrances and numerous biologically active compounds [3]. For a long time, traditional condensation of amines with carbonyl compounds was regarded as the simplest way to prepare imines [4-6]. Highly reactive nature of aldehydes made handling difficult. This was overcome later by replacing aldehydes with alcohols and temporarily producing aldehydes in-situ within the reaction mixture and the subsequent reaction with an amine yield the imine compound [7,8]. Wang and co-workers have recently developed organosilicon supported $\mathrm{TiO}_{2}$ catalyst for this reaction at $160{ }^{\circ} \mathrm{C}$ with added base to achieve a good imine yield [9]. However, these processes yield range of by-products and greatly affect the selectivity to the desirable product.

Amines are easily accessible compounds that can also be attractive precursors to synthesize imine by controlled oxidation. Until recently, several methods for oxidation of secondary amines to imines have been developed, while little attention has been devoted to the oxidation of primary amines. This is probably because of the generated imines, in which $\alpha$-amino hydrogen is present, are generally intermediate products that are rapidly dehydrogenated to nitriles [10-12]. Subsequent research in the area has been dominated by the development of new catalytic processes that allow the aerobic oxidation of primary amines to imines under mild conditions. Angelici and co-workers reported aerobic oxidative homocoupling of primary amines to give imines, catalyzed by gold powder $(50 \mu \mathrm{m}$ size $)$ and gold nanoparticles supported on alumina $\left(5 \% \mathrm{Au} / \mathrm{Al}_{2} \mathrm{O}_{3}\right)$ in toluene at $100{ }^{\circ} \mathrm{C}$ [13]. Following this study $\mathrm{Au} / \mathrm{C}$ and $\mathrm{CuCl}$ catalysts have been employed in this reaction at $100{ }^{\circ} \mathrm{C}$ under molecular oxygen atmosphere $[14,15]$. It is becoming increasingly important to look for new materials that can catalyze reactions under moderate conditions (relatively low temperature and pressure). In this regard, the utilization of sunlight as an energy source to reduce the working temperature has recently attracted much attention [16-18]. Wang and co-workers have attained imines in excellent yields using mesoporous graphite carbon nitride photocatalyst at $80{ }^{\circ} \mathrm{C}$ [19]. In general, unavailability of structurally diverse amines has hampered the synthetic scope of oxidative coupling of benzylic amines to yield corresponding imines, yet this method is highly selective for imines.

Over the last few years, many efforts have been extended to organic redox-transformation reactions using $\mathrm{TiO}_{2}$ photocatalysis [20-25]. However, to date, most of the reported reactions for the synthetic transformations using $\mathrm{TiO}_{2}$ photocatalysts were carried out under UV irradiation and were usually associated with low selectivity [26,27]. Performing visible light induced selective transformations by photocatalysts is a challenge that has gained increasing attention. Recent discoveries demonstrated the surface modification of $\mathrm{TiO}_{2}$ with noble metal complexes or nanoparticles rather than bulk doping might be a better strategy in light of new visible light responsive photocatalysts that could enhance the design of efficient redox reactions under visible light irradiation. Zhao and co-workers achieved this conversion of primary amine to imine with $\mathrm{TiO}_{2}$ under UV light irradiation (>300 nm) [28] and later they also found it is also possible for this reaction to be initiated by visible light irradiation of $\lambda>420 \mathrm{~nm}$ [29]. According to them, amine molecules adsorbed onto $\mathrm{TiO}_{2}$ forms a surface complex that could absorb visible light and so initiate electron transfer and ensuing reactions. Because the 
reaction takes place on the $\mathrm{TiO}_{2}$ surface, we envisioned that ultrafine $\mathrm{TiO}_{2}$ powders with large specific surface areas should exhibit better catalytic activity. A feasible approach to stabilizing $\mathrm{TiO}_{2}$ nanocrystals is to disperse them in an inorganic medium, such as layered clays creating porous composite structures, while ensuring that most of the surface of $\mathrm{TiO}_{2}$ crystals is accessible to various molecules [30]. Nevertheless, the structure of the composite solids has a profound impact on their catalytic performance [31]. The mesoporous composites of anatase nanocrystals and silicate are the catalyst materials of the optimal structure for high photocatalytic activity. Synthetic layered clay, laponite, can be used in the synthesis of the composite as silicate source [30].

Here in we report $\mathrm{TiO}_{2}$ nanocrystal-silicate composite, prepared using laponite, as feasible photocatalyst for the selective oxidation of benzylamine to $N$-benzylidene benzylamine with excellent conversion and selectivity under the irradiation of visible light $(\lambda>460 \mathrm{~nm})$. It is found that in the $\mathrm{TiO}_{2}$-silicate composite catalyst $\mathrm{TiO}_{2}$ is in anatase phase. Reference reactions with anatase show that under identical conditions $\mathrm{TiO}_{2}$-silicate composite catalyst exhibited a much more superior catalytic activity to pure $\mathrm{TiO}_{2}$ (anatase) powder. Nitrogen adsorption data confirms the large surface area of the composite catalyst. Furthermore, we also found that water can be used as the solvent. This catalyst could be employed for heterocoupling of two structurally diverse amines in the synthesis of imines as well as homocoupling of benzylic amines to imines, and the catalyst can be recycled up to five rounds without any significant loss of activity.

\section{Results and Discussion}

The aerobic photocatalytic oxidation of benzylamine to $N$-benzylidene benzylamine was chosen as the model reaction to optimize the reaction system. Reactions are carried out using $500 \mathrm{~W}$ halogen lamps where the light emitted is in $400-800 \mathrm{~nm}$ range. According to the data given in Table 1 it is apparent that $\mathrm{TiO}_{2}$-silicate (abbreviated as $\mathrm{TiO}_{2}-\mathrm{S}$ hereafter) is the most photocatalytically active photocatalyst towards this transformation. Catalyst samples were also prepared by loading $\mathrm{Au}$ and $\mathrm{Pd}$ nanoparticles (NPs) (3\% by weight) and another sample with AuPd alloy NPs $(1.5 \%$ weight of each metal) loaded on to $\mathrm{TiO}_{2}-\mathrm{S}$ composite material (Characterization is provided in $\mathrm{SI}$ ). We observed a lower imine product yield of $60 \%$ with $\mathrm{Au} @ \mathrm{TiO}_{2}-\mathrm{S}$, compared to $82 \%$ by $\mathrm{TiO}_{2}-\mathrm{S}$, despite the enhanced light absorption by Au NPs in the visible region due to localized surface plasmon resonance (LSPR) effect which is characterized by an intense band around $520 \mathrm{~nm}$ (Figure S1, Supplementary Information) [16-18]. This observation also serves as an example to support the fact, light absorption by a material is not the sole governing factor deciding catalysts ability to drive a particular chemical reaction under light irradiation. It appears that the reaction takes place on the surface of anatase, the loaded $\mathrm{Au}$ NPs lower the exposed surface area of $\mathrm{TiO}_{2}$, the catalytically active sites of this system, lowering the accessibility to the reactants. $\mathrm{Pd} @ \mathrm{TiO}_{2}-\mathrm{S}$ catalyst had similar activity to that of $\mathrm{TiO}_{2}-\mathrm{S}$, whereas $\mathrm{AuPd} @ \mathrm{TiO}_{2}-\mathrm{S}$ was slightly sluggish. Results further indicate the unique potential of $\mathrm{TiO}_{2}$ based materials towards oxidation reactions and importance of evaluation of surface modifications of $\mathrm{TiO}_{2}$ for activity improvements. In control experiments, the reaction did not proceed without a photocatalyst or in the dark. 
Table 1. Photocatalytic oxidation of benzylamine to $N$-benzylidene benzylamine over different catalyst materials and solvents. ${ }^{\text {a }}$

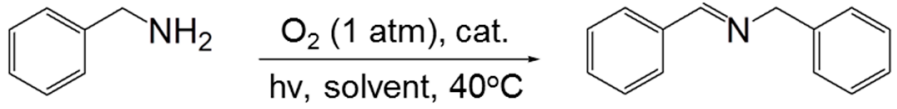

\begin{tabular}{|c|c|c|c|c|c|}
\hline Entry & Catalyst & Solvent & Conv. $(\%)^{b}$ & Sel. $(\%)^{b}$ & Yield (\%) \\
\hline 1 & $\mathrm{TiO}_{2}-\mathrm{S}$ & Acetonitrile & 88 & 92 & 81 \\
\hline 2 & $\mathrm{TiO}_{2}-\mathrm{S}$ & DMSO & 18 & 100 & 18 \\
\hline 3 & $\mathrm{TiO}_{2}-\mathrm{S}$ & THF & 94 & 73 & 69 \\
\hline 4 & $\mathrm{TiO}_{2}-\mathrm{S}$ & Toluene & 74 & 97 & 72 \\
\hline 5 & $\mathrm{Au} @ \mathrm{TiO}_{2}-\mathrm{S}$ & Acetonitrile & 65 & 93 & 60 \\
\hline 6 & $\mathrm{AuPd} @ \mathrm{TiO}_{2}-\mathrm{S}$ & Acetonitrile & 88 & 90 & 79 \\
\hline 7 & $\mathrm{Pd} @ \mathrm{TiO}_{2}-\mathrm{S}$ & Acetonitrile & 89 & 96 & 85 \\
\hline 8 & Laponite & Acetonitrile & 0 & -- & 0 \\
\hline 9 & $\mathrm{TiO}_{2}$ (anatase) & Acetonitrile & 51 & 100 & 51 \\
\hline 10 & H-titanate & Acetonitrile & 73 & 97 & 71 \\
\hline
\end{tabular}

a Reaction conditions: $50 \mathrm{mg}$ catalyst, $0.5 \mathrm{mmol}$ benzylamine, $5 \mathrm{~mL}$ solvent, $500 \mathrm{~W}$ halogen lamp (cut off wavelength below $400 \mathrm{~nm}$ ) intensity $0.36 \mathrm{~W} / \mathrm{cm}^{2}, 1$ atm $\mathrm{O}_{2}, 24 \mathrm{~h} .{ }^{\mathrm{b}}$ Determined by GC analysis. $\mathrm{DMSO}=$ dimethyl sulfoxide, $\mathrm{THF}=$ tetrahydrofuran.

As can be seen in Table 1, activity of $\mathrm{TiO}_{2}-\mathrm{S}$ is superior to that of an equivalent amount of $\mathrm{TiO}_{2}$ (anatase) as the photocatalyst material. In order to understand this change in behavior we closely studied the light absorption abilities of both $\mathrm{TiO}_{2}$ (anatase) and $\mathrm{TiO}_{2}-\mathrm{S}$, in the presence and absence of benzylamine. UV-Visible diffuse reflectance spectra of benzylamine adsorbed $\mathrm{TiO}_{2}$ (anatase) and $\mathrm{TiO}_{2}-\mathrm{S}$ shows increased absorbance compared to solitary $\mathrm{TiO}_{2}$ (anatase) and $\mathrm{TiO}_{2}-\mathrm{S}$, particularly in the visible region. This observation agrees well with previous reports, where electron rich molecules like amines make a charge transfer complex with $\mathrm{TiO}_{2}$ and respond to visible light illumination [29]. It is also notable, the absorption of benzylamine adsorbed on $\mathrm{TiO}_{2}-\mathrm{S}$ is significantly high compared to benzylamine adsorbed on $\mathrm{TiO}_{2}$ (anatase) as shown in the Figure $1 \mathrm{~A}$. Even though $\mathrm{TiO}_{2}$ is present in anatase phase in both $\mathrm{TiO}_{2}$ and $\mathrm{TiO}_{2}-\mathrm{S}$ photocatalysts used in the current study, the distribution of anatase particles is different in $\mathrm{TiO}_{2}-\mathrm{S}$. During $\mathrm{TiO}_{2}-\mathrm{S}$ preparation, layered clay structure of precursor material laponite clay is lost as a result of the acidic titanium sol solution reacting with hydroxyl groups in the clay layers that are bound to magnesium ions within the layer [31]. Most of the magnesium in the clay was leached out in this way. Composition of the catalyst estimated by energy dispersive X-ray (EDX) confirms high weight percentage of silicate in the composite catalyst despite the leaching of Mg units. During preparation Si:Mg ratio (by weight) decreased from 1:0.58 to 1:0.25, this together with TEM image is a clear indication that ordered layer structure is damaged. $\mathrm{Thus} \mathrm{TiO}_{2}$ in this $\mathrm{TiO}_{2}-\mathrm{S}$ composite catalyst exists as discrete anatase crystals on fragmentized pieces of silicate. Correspondingly this composite structure restrains agglomeration of anatase particles leading to high exposed surface area of $\mathrm{TiO}_{2}$. Brunauer-Emmett-Teller (BET) surface area of initial laponite clay changed from $330.6 \mathrm{~m}^{2} \mathrm{~g}^{-1}$ to $518.3 \mathrm{~m}^{2} \mathrm{~g}^{-1}$ in the final $\mathrm{TiO}_{2}-\mathrm{S}$ catalyst material. The composite has porosity of about $0.4 \mathrm{~cm}^{3} / \mathrm{g}$ and a mean pore size of $5 \mathrm{~nm}$. Finally, in the obtained composite catalyst silica particles and anatase crystals exist as inter-dispersed phases in nanometer scale with a highly porous 
structure as can be seen in Figure 1B. X-ray diffraction (XRD) pattern of the catalyst only exhibit peaks responsible for the anatase phase of $\mathrm{TiO}_{2}$ with no peaks related to silicate units or laponite clay, this indicates silica is present in the amorphous phase, and anatase particles of mean crystal size $4.22 \mathrm{~nm}$ (estimated by Debye-Scherrer equation using the broadening of the highly intense (101) XRD peak at $2 \theta=25.3^{\circ}$ ) have homogeneously crystallized over amorphous silica moiety. This $\mathrm{TiO}_{2}-\mathrm{S}$ structure obtained in the present study, offers high thermal and chemical stability, also provides ample opportunity for the reactant molecules to interact with energetic charge carriers. Smaller anatase particles reduce the possibility of charge recombination, since charge carriers are generated at the close proximity of surface and efficiently captured by benzylamine and oxygen molecules on the surface.
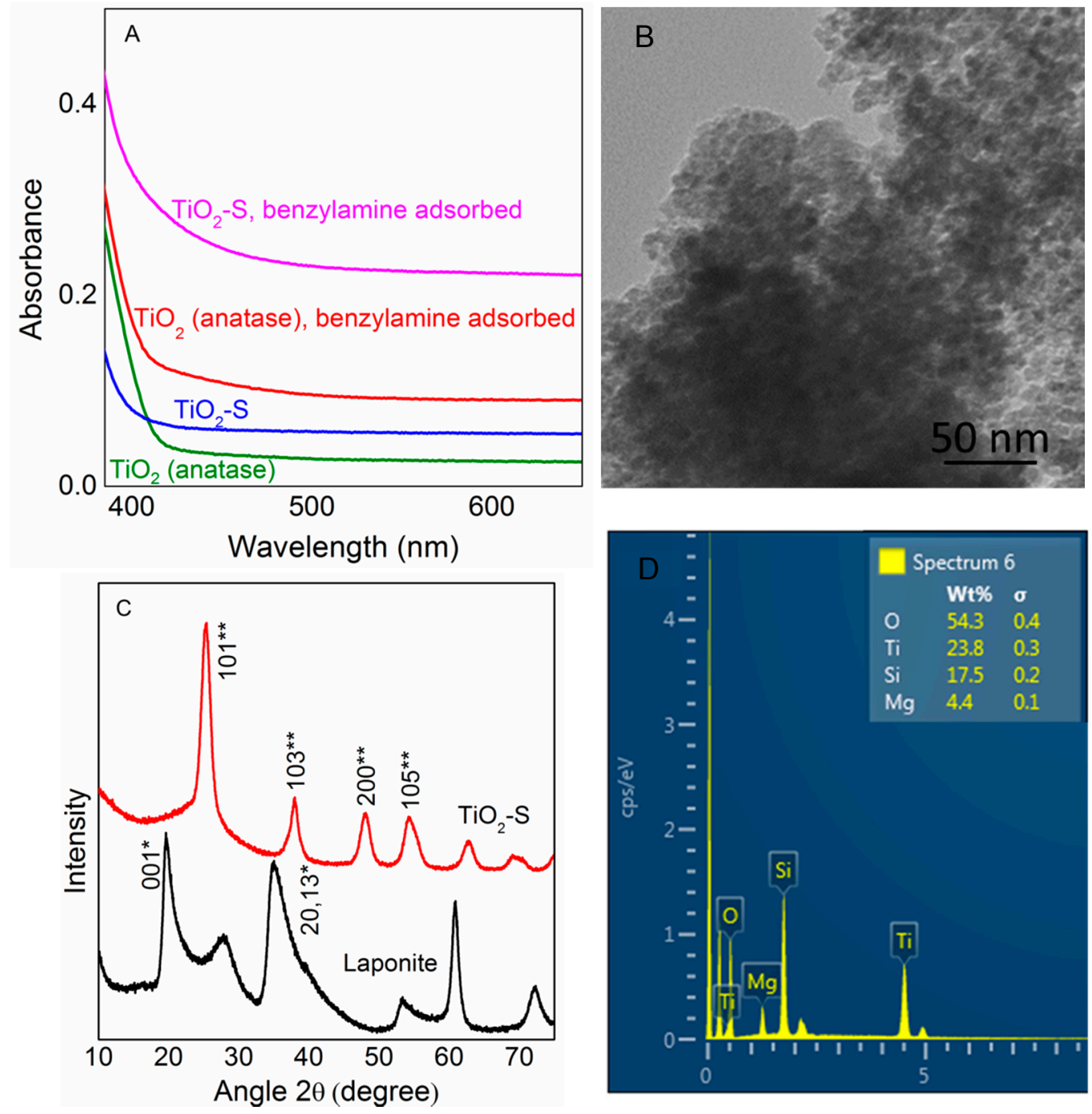

Figure 1. (A) UV-Visible diffuse reflectance spectra; (B) transmission electron microscopy (TEM) image; (C) X-ray diffraction (XRD) peak patterns indexed for *_-silicate phase and **_anatase phase; (D) energy dispersive X-ray (EDX) spectra of $\mathrm{TiO}_{2}-\mathrm{S}$ composite catalyst.

To further investigate the contribution from light in this reaction, we conducted a series of reactions at variable intensities (Figure $2 \mathrm{~A}$ ). The conversion rate of benzylamine on $\mathrm{TiO}_{2}-\mathrm{S}$ catalyst increased gradually as the intensity increased, with the other reaction conditions unchanged (Experimental 
section). Selectivity to the product imine had a little influence on the intensity; however, overall imine yield (conversion rate $\mathrm{x}$ selectivity) increased with the intensity. Such a tendency reveals a strong dependence on the intensity for the light induced oxidative coupling of benzylamine, because in general light incident with a higher intensity is able to generate more energetic charge carriers (holes and electrons). Such conditions favor stronger interaction between benzylamine and the catalyst, and positively influence the reaction.
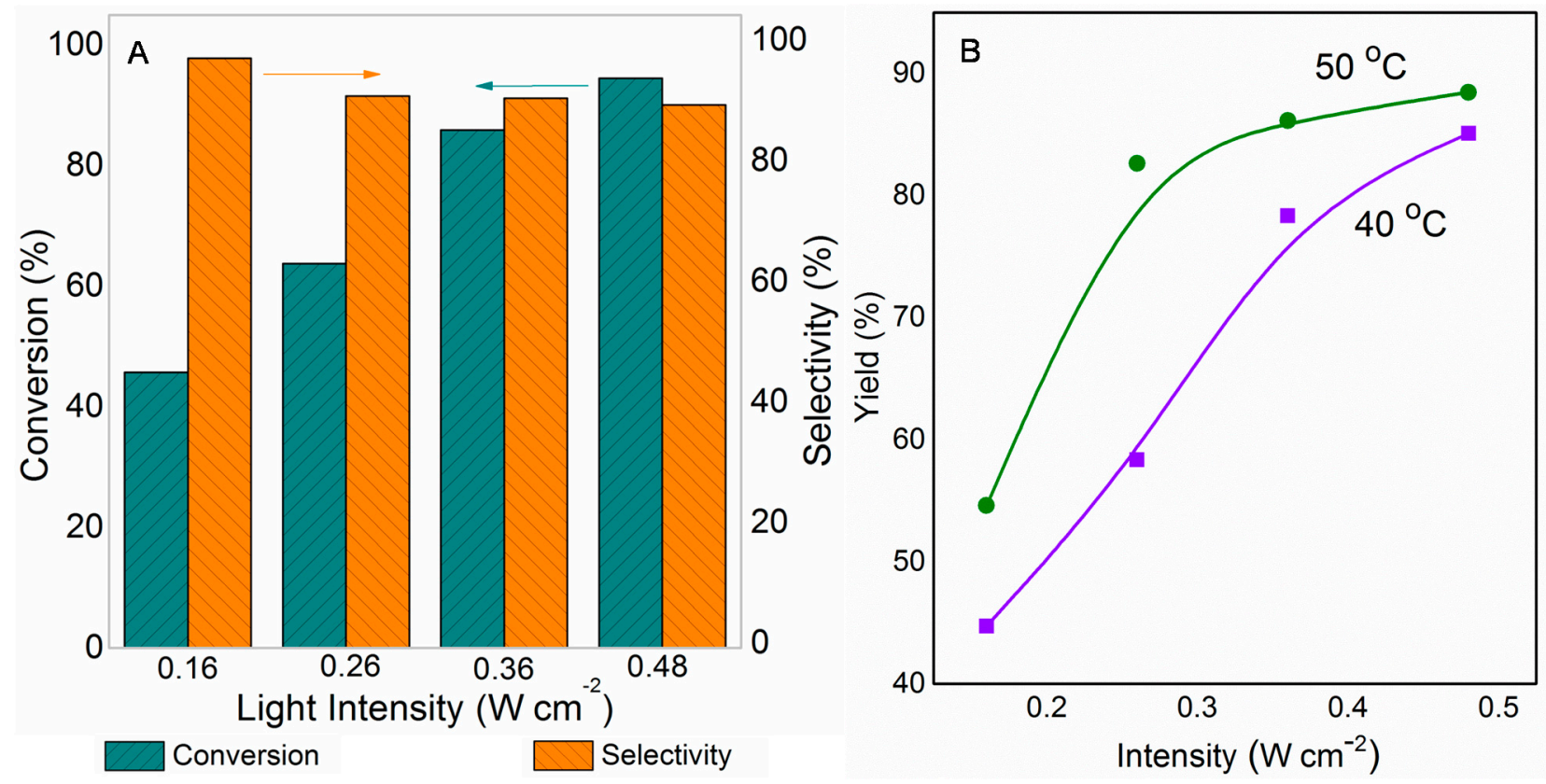

Figure 2. The effect of (A) light intensity and (B) temperature influence of the irradiation on the oxidative coupling of benzylamine.

Impact of the temperature on the yield of the reaction was studied by conducting the experiments at two different temperatures slightly above room temperature $\left(40{ }^{\circ} \mathrm{C}\right.$ and $\left.50^{\circ} \mathrm{C}\right)$. As shown in Figure $2 \mathrm{~B}$, observed yield was always high for the reaction under the study, oxidative coupling of benzylamine to imine at higher temperature for a given constant intensity within 40 to $50{ }^{\circ} \mathrm{C}$ temperature range. For instance, at $40{ }^{\circ} \mathrm{C}$ and $0.26 \mathrm{~W} \cdot \mathrm{cm}^{-2}$ intensity, $58 \%$ of benzylamine was converted to imine product whereas at $50{ }^{\circ} \mathrm{C}$ it was $83 \%$. At high temperature however, the enhancement in the yield by increasing the intensity lessens since the selectivity to the imine product starts to decline, though reaction proceeds at a higher conversion rate. As the intensity was increased, the difference between the yields at 40 and $50{ }^{\circ} \mathrm{C}$ finally decreased, even though higher conversion rate was observed for $50{ }^{\circ} \mathrm{C}$ compared to that at $40{ }^{\circ} \mathrm{C}$ in all cases.

The dependence of yield on the irradiation wavelength was studied using five monochromatic light emitting diodes (LEDs) and it shows that higher photocatalytic yields are achieved under irradiation of short wavelengths $(<460 \mathrm{~nm})$. Anatase phase of $\mathrm{TiO}_{2}$ exhibits a band gap of $3.2 \mathrm{eV}(387.5 \mathrm{~nm})$ where as in this case $\mathrm{TiO}_{2}-\mathrm{S}$ is highly active up to $460 \mathrm{~nm}$. Figure 3 demonstrates the apparent quantum yield (A.Q.Y) dependence on the incident wavelength; A.Q.Y. is a measure of imine yield per photon of energy absorbed per unit time. This finding indicates that composite $\mathrm{TiO}_{2}-\mathrm{S}$ catalyst structure has a broad light response below $460 \mathrm{~nm}$ in the visible region due to the collective effects of benzylamine 
adsorbed $\mathrm{TiO}_{2}$ (anatase) charge transfer surface complex and high population of defects in the composite photocatalyst structure. This reveals that $\mathrm{TiO}_{2}-\mathrm{S}$ catalyst can function at a lower cut-off edge $\left(460 \mathrm{~nm}\right.$ ) compared to $420 \mathrm{~nm}$ cut-off for solitary $\mathrm{TiO}_{2}$ (anatase) system reported by Zhao and co-workers [29]. It is noteworthy that, in the composite structure of the catalyst there are contacts between silicate and anatase crystals. At these sites, the anatase surface is similar to the silica doped anatase surface that exhibits light absorption and visible light photocatalytic activity [32].

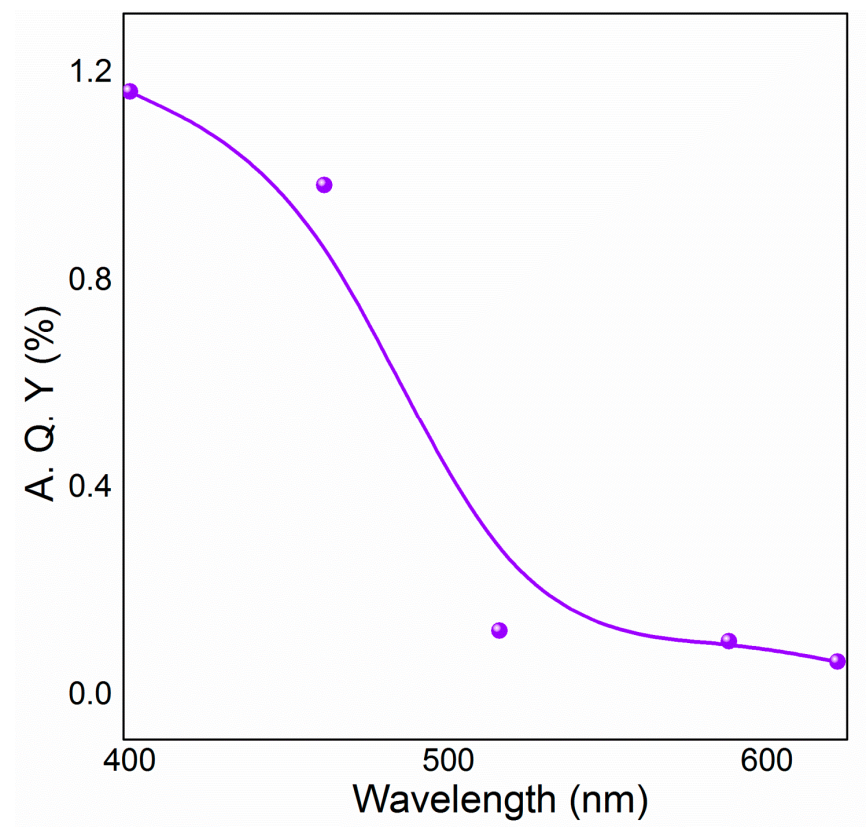

Figure 3. The effect of wavelength of the irradiation on the oxidative coupling of benzylamine.

According to the results summarized in Table 1, the solvent has important impact on the catalytic activity. Acetonitrile serves as the best solvent for this reaction while the poorest performance was observed in DSMO. Water is a viable solvent for organic reactions, and it is interesting to study the impact of water as the reaction medium. For some organic reactions, water exhibits special reactivity or selectivity due to its unique physical properties. In the present study, benzylamine oxidation reaction proceeded in water at a moderate conversion rate of $45.6 \%$ under the illumination of $500 \mathrm{~W}$ halogen lamp (400-800 nm) for $24 \mathrm{~h}$, but with an excellent selectivity where the sole product being $\mathrm{N}$-benzylidene benzylamine with an overall yield of $46 \%$. Doubling the amount of catalyst from $50 \mathrm{mg}$ to $100 \mathrm{mg}$ of $\mathrm{TiO}_{2}-\mathrm{S}$ enhanced the reaction yield to $62 \%$ without compromising the selectivity.

Motivated by this result, we expanded the scope of the substrates for the oxidation of amines to imines. Table 2 summarizes the photocatalytic oxidation of the benzylic amines to corresponding imines with their conversion rate and selectivity. Oxidation of primary benzylic amines substituted with an electron donating group (Table 2, entry 2-3) proceeded efficiently under visible light irradiation with good to high conversion rates and high selectivity for the imine product. Substituent group influences the conversion rate of the reaction than the selectivity to the imine product. The relatively low conversion rate for the oxidative coupling of 4-chlorobenzylamine (Table 2, entry 4) into its corresponding imine might be caused by inductive effects of $\mathrm{C}-\mathrm{Cl} \sigma$-bond polarity. No change was observed when aniline was subjected to the reaction, this is consistent with our hypothesis that the 
presence of a $-\mathrm{H}$, bonded to the $\alpha$-carbon is important for this transformation to take place. Furthermore, non-aromatic cyclic amines (Table 2, entry 7-8) did not yield the desired imine product. Control experiments carried out using cyclohexylamine (consist of a single $\alpha$-hydrogen) produced the corresponding oxime (cyclohexanone oxime) instead of the imine. Benzaldehyde oxime was one of the products observed during the time course of the reaction of benzylamine.

Table 2. Aerobic oxidation of primary benzylic amines photocatalyzed by $\mathrm{TiO}_{2}-\mathrm{S}$ under visible light irradiation. ${ }^{\text {a }}$<smiles>[R]c1ccc(C=NCc2ccc([R])cc2)cc1</smiles>

\begin{tabular}{|c|c|c|c|c|c|}
\hline Entry & Substrate & Product & Con. $(\%)^{b}$ & Select. $(\%)^{b}$ & Yield (\%) \\
\hline 1 & & & 88 & 92 & 81 \\
\hline 2 & & & 96 & 92 & 88 \\
\hline 3 & & & 100 & 96 & 96 \\
\hline 4 & & & 57 & 100 & 57 \\
\hline & & & $88^{\mathrm{c}}$ & $>99^{\mathrm{c}}$ & $87^{c}$ \\
\hline 5 & & & 91 & 35 & 32 \\
\hline 7 & & & 51 & 20 & 10 \\
\hline 8 & & & 74 & 48 & 36 \\
\hline
\end{tabular}

${ }^{a}$ Reaction condition: $50 \mathrm{mg}$ catalyst, $0.5 \mathrm{mmol}$ amine substrate, $5 \mathrm{~mL}$ acetonitrile, $500 \mathrm{~W}$ halogen lamp (cut-off wavelength below $400 \mathrm{~nm}$ ) intensity $0.36 \mathrm{~W} / \mathrm{cm}^{2}, 1$ atm $\mathrm{O}_{2}, 24 \mathrm{~h} .{ }^{\mathrm{b}}$ Determined by GC analysis. ${ }^{\mathrm{c}}$ Reaction time $36 \mathrm{~h}$.

This reaction goes through the widely known intermediate benzaldehyde and a tentative mechanistic pathway is given in Scheme 1 based on the products observed. The photocatalysts contribution is mainly in the step of benzylamine oxidation to benzaldehyde, whereas the condensation of benzaldehyde with a benzylamine molecule leading to the imine product is faster. In the oxidation step, $\mathrm{TiO}_{2}$-benzylamine surface complex absorb visible light (400-800 nm) and excite electrons. These excited electrons are then captured by oxygen molecules adsorbed on $\mathrm{TiO}_{2}$ surface, then in the proceeding steps oxygen interacts with benzylamine and the substrate molecules lose the $\mathrm{H}$ bonded to the $\alpha$-carbon atom, and oxidized to aldehyde. Thus, it is rational that under visible light irradiation, the oxygen molecules adsorbed on the catalyst capture the light excited electrons, and react with the $\mathrm{H}$ at the $\alpha$-carbon. Role of oxygen is further confirmed, when the reaction was carried out in the air atmosphere benzylamine exhibit a relatively lower observed conversion rate of $51 \%$ and a selectivity of $96 \%$, yielding $49 \%$ of imine after $24 \mathrm{~h}$. This mechanism agrees well with the observed product selectivity results. At higher conversions of benzylamine, a decrease in the selectivity for the imine 
occurs and benzaldehyde appears in the products. This is due to the fact that, increased consumption of benzylamine in the solution could not ensure the complete condensation of aldehyde and the amine.

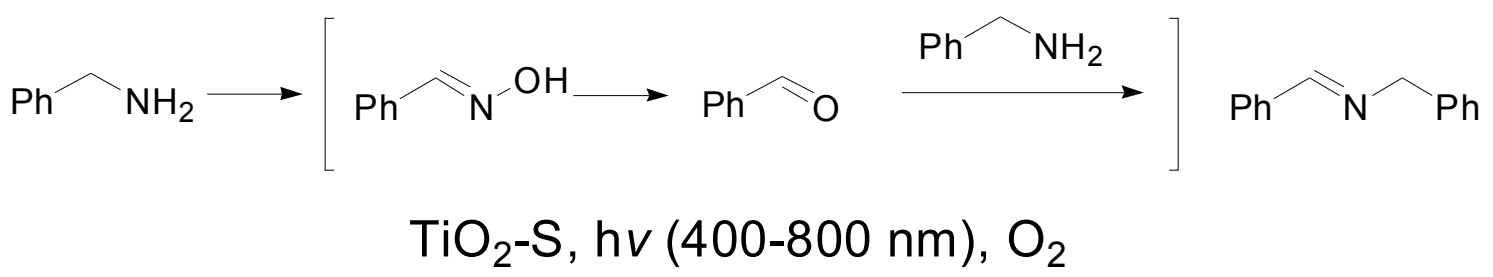

Scheme 1. Tentative reaction pathway.

Ability of $\mathrm{TiO}_{2}-\mathrm{S}$ photocatalyst to catalyze the oxidative cross-coupling of two benzylic amines with different substituent groups to yield a heterocoupled imine product was also studied using benzylamine, 4-methylbenzylamine and 4-methoxybenzylamine (two at a given reaction). Results demonstrated a poor selectivity since all four possible imines were observed in relatively similar yields after $24 \mathrm{~h}$. Oxidative coupling of benzylamine with 4-methylbenzylamine had $95 \%$ of imine product yield. Self-coupling products of benzylamine (28\%) and 4-methylbenzylamine (22\%) were observed together with the two heterocoupled imines $(50 \%)$, the product distribution of heterocoupling of benzylamine with 4-methylbenzylamine is as desired $(\sim 1: 1: 1: 1)$ since difference in the nucleophilicities of " $\mathrm{H}$ " and methyl group is not significant. In order to evaluate the product distribution over the time span of this heterocoupled imine synthesis, we chose benzylamine and 4-methoxybenzylamine as the two benzylic amine substrates and the reaction profile is given in the Table 3, this reveals both the precursor imines produce the corresponding aldehydes as per the oxygenation step shown in Scheme 1, and then reacts with a free amine molecule to yield the final imine. Aldehyde of more electro deficient nucleus reacts faster with the more electron rich amine (P3) at early stages of the reaction and then with either amine as the reaction is progressing. Rate of aldehyde formation is slower in electron rich benzene nucleus, benzylamine in this system and it acts as the nucleophile (amine half), while 4 methoxybezylamine is easier to oxidize and preferentially be the aldehyde half. In the product distribution more P3 and P4 are observed during the whole cause of the reaction indicating high formation and reactivity of 4-methoxybenzaldehyde. Dual amine systems of benzylamine/aniline and 4-methoxybenzylamine/aniline yield only the self-coupled imines of benzylamine (92\%) and 4-methoxybenzylamine (96\%) respectively. Amount of aniline introduced in the reaction system remained unchanged even after the reaction, portraying its inert role in this photocatalyzed oxidative coupling reaction, anilne with a $-\mathrm{NH}_{2}$ unit in its structure failed to participate in this heterocoupling reactions at least as the amine half.

Reusability of the catalyst is an important parameter in heterogeneous catalysis. The composite $\mathrm{TiO}_{2}-\mathrm{S}$ photocatalyst studied in this system can be recovered readily from aqueous or organic solutions by simple filtration or sedimentation. The anatase nanocrystals in these composite samples are linked to silicate pieces such that grains in the $\mu \mathrm{m}$ scale are formed. Operational life of this catalyst examined over five consecutive rounds (Figure 4) revealed no apparent activity loss after five rounds. This further confirms the thermal and chemical stability of the catalyst. However selectivity towards the imine product was gradually decreased during each cycle lowering the overall product yield. 
Table 3. Time conversion plot for oxidative coupling of benzylamine with 4-methoxybenzylamine ${ }^{\text {a }}$.

\begin{tabular}{cccccccc}
\hline \multirow{2}{*}{ Entry } & \multirow{2}{*}{ Time (h) } & \multicolumn{3}{c}{ Conversion (\%) } & \multicolumn{4}{c}{ Selectivity (\%) } \\
\cline { 2 - 8 } & & Benzylamine & 4-Methoxybenzylamine & P1 & P2 & P3 & P4 \\
\hline 1 & 2 & 13 & 13 & 0 & 0 & 100 & 0 \\
2 & 4 & 41 & 57 & 12 & 12 & 40 & 36 \\
3 & 8 & 84 & 90 & 15 & 16 & 38 & 32 \\
4 & 17 & 96 & 97 & 19 & 16 & 40 & 26 \\
5 & 20 & 96 & 97 & 21 & 16 & 40 & 24 \\
\hline
\end{tabular}

a Reaction Conditions: $25 \mathrm{mg}$ catalyst, $0.25 \mathrm{mmol}$ amine substrates, $2 \mathrm{~mL}$ acetonitrile, $500 \mathrm{~W}$ halogen lamp (cut-off wavelength below $400 \mathrm{~nm}$ ) intensity $0.36 \mathrm{~W} / \mathrm{cm}^{2}, 1 \mathrm{~atm} \mathrm{O}_{2}$.

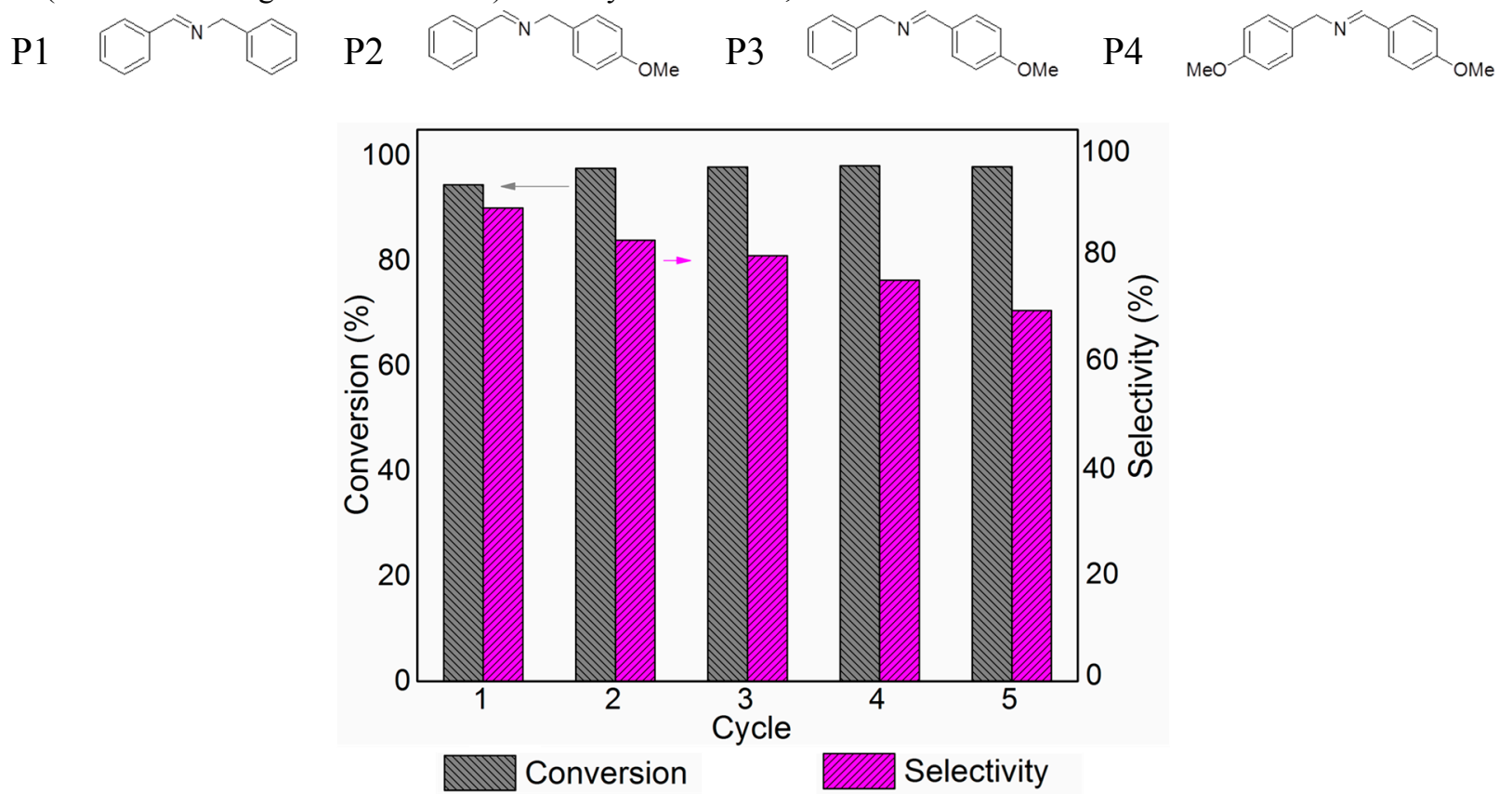

Figure 4. Reusability data of $\mathrm{TiO}_{2}-\mathrm{S}$ catalyst for the oxidative coupling of benzylamine.

\section{Experimental Section}

\subsection{General Information and Materials}

The laponite clay was supplied from Fernz specialty chemicals Australia, all other chemicals were purchased from Sigma Aldrich (Castle Hill NSW, Australia) and used as received without further purification. Water used in all experiments was milli-Q water passing through an ultra-purification system.

\subsection{General Procedure for the Synthesis of $\mathrm{TiO}_{2}-\mathrm{S}$ Composite}

$\mathrm{TiO}_{2}$ precursor was prepared by hydrolyzing $\mathrm{Ti}\left(\mathrm{OCH}_{3}\right)_{4}$ in $\mathrm{HCl}$ for $3 \mathrm{~h}$ following a slightly modified method proposed by J. Sterte [30,31,33].

Initially $1.0 \mathrm{~g}$ of laponite was slowly dispersed in $50 \mathrm{~mL}$ of deionized water and kept stirring until it was transparent. Then $4.0 \mathrm{~g}$ of polyethylene glycol (FW 585) surfactant and the metal precursor solution was added drop wise with continuous stirring. Mixture was then transferred to teflon covered autoclaves and heated at $100{ }^{\circ} \mathrm{C}$ for 2 days. The solid was then recovered from centrifugation, followed 
by washing with water until no more chloride ions left (confirmed by a test with $\mathrm{AgNO}_{3}$ ). Product was then dried in air and finally calcined at $500{ }^{\circ} \mathrm{C}$ for $20 \mathrm{~h}$ with the step being $2{ }^{\circ} \mathrm{C} \cdot \mathrm{min}^{-1}$.

\subsection{Characterization of $\mathrm{TiO}_{2}-\mathrm{S}$ Composite}

The diffuse reflectance UV/Vis (DR-UV/Vis) spectra were recorded on a Cary 5000 UV/Vis-NIR Spectrophotometer (Agilent, Santa Clara, CA, USA). X-ray diffraction (XRD) patterns of the samples were recorded on a Philips PANalytical X'Pert PRO diffractometer (PANalytical, Sydney, Australia) using $\mathrm{CuK} \alpha$ radiation $(1=1.5418 \AA)$ at $40 \mathrm{kV}$ and $40 \mathrm{~mA}$. Transmission electron microscopy (TEM) images were taken with a Philips CM200 Transmission electron microscope (Philips, Eindhoven, The Netherlands) employing an accelerating voltage of $200 \mathrm{kV}$. The specimens were fine powders deposited onto a copper micro grid coated with a holey carbon film. Nitrogen physisorption isotherms were measured on the Tristar II 3020 (Micromeritics, Norcross, GA, USA). Prior to the analysis, sample was degassed at $110{ }^{\circ} \mathrm{C}$ overnight under high vacuum. The specific surface area was calculated by the Brunauer-Emmett-Teller (BET) method from the data in a $\mathrm{P} / \mathrm{P}^{\circ}$ range between 0.05 and 0.2 . The compositional data was determined by energy-dispersive X-ray spectroscopy (EDS) (EDAX, Mahwah, NJ, USA ) attached to an FEI Quanta 200 scanning electron microscope (SEM, Quanta, OR, USA).

\subsection{General Procedure for the Photocatalytic Reactions}

Benzylic amine compound $0.5 \mathrm{mmol}, 5 \mathrm{~mL}$ of solvent were measured to a clean dry reactor tube. $50 \mathrm{mg}$ of the catalyst was added and finally the reactor was purged with oxygen gas. These reactors were kept magnetically stirring in front of a $500 \mathrm{~W}$ halogen lamp (except for dark and wavelength experiments) for $24 \mathrm{~h}$ at $40{ }^{\circ} \mathrm{C}$. At the end of the reaction $1 \mathrm{~mL}$ samples were collected in to small glass vials after filtering out the solid catalyst using $0.2 \mu \mathrm{m}$ milli pore filter. We tested for the products using a gas chromatograph (GC, Agilent, Santa Clara, CA, USA) equipped with a DB 5 column. For wavelength experiments, 5 monochromatic light emitting diodes (LEDs) of 390-410 nm, 460-462 nm, $515-517 \mathrm{~nm}, 587.5-560 \mathrm{~nm}$ or 620-625 nm was used.

\section{Conclusions}

We have successfully applied $\mathrm{TiO}_{2}-\mathrm{S}$ composite photocatalyst in the oxidative coupling of benzylamine to imine under visible light irradiation. The numerous contacts between the anatase crystals and silicate and high population of defects in the composite photocatalyst are the possible reasons behind the enhanced visible light activity. The formation of imines proceed via an oxidation pathway: under visible light irradiation, the oxygen molecules adsorbed on the catalyst capture the light excited electrons, and react with the $\mathrm{H}$ bonded to the $\alpha$-carbon of the substrate molecules, which is oxidized to aldehyde. The condensation of the aldehyde with amine yields the product imine. This photocatalyst has a very high activity in the region $\lambda>460 \mathrm{~nm}$. This range is much broader compared to previously reported results for anatase materials $(\lambda>420 \mathrm{~nm})$. Intensity, wavelength and reaction temperature can be tuned to optimize the reaction rate of $\mathrm{TiO}_{2}-\mathrm{S}$ catalyzed oxidative coupling of benzylic amines. Water can be used as a solvent giving moderate conversion rate but sole product. 
These findings encourage us to further study the surface modified titania based materials for selective organic synthesis.

\section{Supplementary Materials}

Supplementary materials can be accessed at: http://www.mdpi.com/1420-3049/20/02/1941/s1.

\section{Acknowledgments}

This project was supported by Australian Research Council (ARC DP110104990).

\section{Author Contributions}

S.Z. and H.Z. designed the research; S.Z. performed the research, analysed the data and wrote the paper. Both authors read and approved the final manuscript.

\section{Conflicts of Interest}

The authors declare no conflict of interest.

\section{References}

1. Kobayashi, S.; Mori, Y.; Fossey, J.S.; Salter, M.M. Catalytic Enantioselective Formation of C-C Bonds by Addition to Imines and Hydrazones: A Ten-Year Update. Chem. Rev. 2011, 111, 2626-2704, doi:10.1021/cr100204f.

2. Adams, J.P. Imines, Enamines and Oximes. J. Chem. Soc. Perkin Trans. 1 2000, 125-139, doi:10.1039/A808142E.

3. Largeron, M. Protocols for the Catalytic Oxidation of Primary Amines to Imines. Eur. J. Org. Chem. 2013, 24, 5225-5235.

4. Granzhan, A.; Riis-Johannessen, T.; Scopelliti, R.; Severin, K. Combining Metallasupramolecular Chemistry with Dynamic Covalent Chemistry: Synthesis of Large Molecular Cages. Angew. Chem. Int. Ed. 2010, 49, 5515-5518.

5. Belowich, M.E.; Stoddart, J.F. Dynamic Imine Chemistry. Chem. Soc. Rev. 2012, 41, 2003-2024.

6. Rasdi, F.R. M.; Phan, A.N.; Harvey, A.P. Rapid Determination of the Reaction Kinetics of an $N$-butylbenzaldimine Synthesis Using a Novel Mesoscale Oscillatory Baffled Reactor. Procedia Eng. 2012, 42, 1662-1675.

7. Alessandro, Z.; Jose, A.M.; Eduardo, P. One-Pot Preparation of Imines from Nitroarenes by a Tandem Process with an Ir-Pd Heterometallic Catalyst. Chem. Eur. J. 2010, 16, 10502-10506.

8. Kwon, M.S.; Kim, S.; Park, S.; Bosco, W.; Chidrala, R.K.; Park, J. One-Pot Synthesis of Imines and Secondary Amines by Pd-Catalyzed Coupling of Benzyl Alcohols and Primary Amines. J. Org. Chem. 2009, 74, 2877-2879.

9. Wang, H.; Zhang, J.; Cui, Y.M.; Yang, K.F.; Zheng, Z.J.; Xu. L.W. Dehydrogenation and Oxidative Coupling of Alcohol and Amines Catalysed by Organosilicon-Supported $\mathrm{TiO}_{2} @$ PMHSIPN. RSC Adv.2014, 4, 34681-34686. 
10. Yamaguchi, K.; Mizuno, N. Efficient Heterogeneous Aerobic Oxidation of Amines by a Supported Ruthenium Catalyst. Angew. Chem. Int. Ed. 2003, 42, 1480-1483.

11. Yamaguchi, K.; Mizuno, N. Scope, Kinetics, and Mechanistic Aspects of Aerobic Oxidations Catalysed by Ruthenium Supported on Alumina. Chem. Eur. J. 2003, 9, 4353-4361.

12. Mizuno, N.; Yamaguchi, K. Selective Aerobic Oxidations by Supported Ruthenium Hydroxide Catalysts. Catal. Today 2008, 132, 18-26.

13. Zhu, B.; Lazar, M.; Trewyn, B.G.; Angelici, R.J. Aerobic Oxidation of Amines to Imines Catalyzed by Bulk Gold Powder and by Alumina-Supported Gold. J. Catal. 2008, 260, 1-6.

14. Grirrane, A.; Corma, A.; Garcia, H. Highly Active and Selective Gold Catalysts for the Aerobic Oxidative Condensation of Benzylamines to Imines and One-Pot, Two-Step Synthesis of Secondary Benzylamines. J. Catal. 2009, 264, 138-144.

15. Patil, R.D.; Adimurthy, S. Copper-Catalyzed Aerobic Oxidation of Amines to Imines under Neat Conditions with Low Catalyst Loading. Adv. Synth. Catal. 2011, 353, 1695-1700.

16. Ke, X.; Zhang, X.; Zhao, J.; Sarina, S.; Barry, J.; Zhu, H. Selective Reductions using Visible Light Photocatalysts of Supported Gold Nanoparticles. Green Chem. 2013, 15, 236-244.

17. Zhu, H.; Ke, X.; Yang, X.; Sarina, S.; Liu, H. Reduction of Nitroaromatic Compounds on Supported Gold Nanoparticles by Visible and Ultraviolet Light. Angew. Chem. Int. Ed. 2010, 122, 9851-9855.

18. Zhang. X.; Ke, X.; Zhu, H. Zeolite-Supported Gold Nanoparticles for Selective Photooxidation of Aromatic Alcohols under Visible-Light Irradiation. Chem. Eur. J. 2012, 18, 8048-8056.

19. Su, F.; Mathews, S.C.; Mohlmann, L.; Antonietti, M.; Wang, X.; Blechert, S. Aerobic Oxidative Coupling of Amines by Carbon Nitride Photocatalysis with Visible Light. Angew. Chem. Int. Ed. 2011, 50, 657-660.

20. Shiraishi, Y.; Hirai, T.; Selective Organic Transformations on Titanium Oxide-Based Photocatalysts. J. Photochem. Photobiol. C 2008, 9, 157-170.

21. Palmisano, G.; Garcia-Lopez, E.; Marci, G.; Loddo, V.; Yurdakal, S.; Augugliaro, V.; Palmosano, L. Advances in Selective Conversions by Heterogeneous Photocatalysis. Chem. Commun. 2010, 46, 7074-7089.

22. Augugliaro, V.; Palmisano, L. Green Oxidation of Alcohols to Carbonyl Compounds by Heterogeneous Photocatalysis. ChemSusChem 2010, 3, 1135-1138.

23. Yurdakal, S.; Palmisano, G.; Loddo, V.; Augugliaro, V.; Palmisano, L. Nanostructured Rutile $\mathrm{TiO}_{2}$ for Selective Photocatalytic Oxidation of Aromatic Alcohols to Aldehydes in Water. J. Am. Chem. Soc. 2008, 130, 1568-1569.

24. Tsukamoto, D.; Ikeda, M.; Shiraishi, Y.; Hara, T.; Ichikuni, N.; Tanaka, S.; Hirai, T. Selective Photocatalytic Oxidation of Alcohols to Aldehydes in Water by $\mathrm{TiO}_{2}$ Partially Coated with $\mathrm{WO}_{3}$. Chem. Eur. J. 2011, 17, 9816-9826.

25. Palmisano, G.; Augugliaro, V.; Pagliaro, M.; Palmisano, L. Photocatalysis: A Promising Route for 21st Century Organic Chemistry. Chem. Commun. 2007, 33, 3425-3437.

26. Fox, M.A.; Dulay, M.T. Heterogeneous Photocatalysis. Chem. Rev. 1993, 93, 341-357.

27. Maldotti, A.; Molinari, A.; Amadelli, R. Photocatalysis with Organized Systems for Oxofunctionalization of Hydrocarbons by $\mathrm{O}_{2}$. Chem. Rev. 2002, 102, 3811-3836. 
28. Li, N.; Lang, X.; Ma, W.; Ji, H.; Chen, C.; Zhao, J.C. Selective Aerobic Oxidation of Amines to Imines by $\mathrm{TiO}_{2}$ Photocatalysis in Water. Chem. Commun. 2013, 49, 5034-5036.

29. Lang, X.; Ma, W.; Zhao, Y.; Chen, C.; Ji, H.; Zhao. J. Visible-Light-Induced Selective Photocatalytic Aerobic Oxidation of Amines into Imines on $\mathrm{TiO}_{2}$. Chem. Eur. J. 2012, 18, 2624-2631.

30. Li, J.; Chen, C.; Zhao, J.; Zhu, H.; Orthman, J. Photodegradation of Dye Pollutants on $\mathrm{TiO}_{2}$ Nanoparticles Dispersed in Silicate under UV-Vis Irradiation. Appl. Catal. B: Environ. 2002, 37, 331-338.

31. Zhu, H.Y.; Zhao, J.C.; Liu, J.W.; Yang, X.Z.; Shen, Y.N. General Synthesis of a Mesoporous Composite of Metal Oxide and Silicate Nanoparticles from a Metal Salt and Laponite Suspension for Catalysis. Chem. Mater. 2006, 18, 3993-4401.

32. Yang, D.; Cheng, C.; Zheng, Z.; Liu, H.; Waclawik, E.R.; Yan, Z.; Huang, Y.; Zhang, H.; Zhao, J.; Zhu, H. Grafting Silica Species on Anatase Surface for Visible Light Photocatalytic Activity. Energy Environ. Sci. 2011, 4, 2279-2287.

33. Sterte, J. Synthesis and Properties of Titanium Oxide Cross-Linked Montmorillonite. Clays Clay Miner. 1986, 34, 658-664.

Sample Availability: Not available.

(C) 2015 by the authors; licensee MDPI, Basel, Switzerland. This article is an open access article distributed under the terms and conditions of the Creative Commons Attribution license (http://creativecommons.org/licenses/by/4.0/). 